\title{
Threshold-based bit error rate for stopping iterative turbo decoding in a varying SNR environment
}

\begin{abstract}
Online bit error rate (BER) estimation (OBE) has been used as a stopping iterative turbo decoding criterion. However, the stopping criteria only work at high signal-to-noise ratios (SNRs), and fail to have early termination at low SNRs, which contributes to an additional iteration number and an increase in computational complexity. The failure of the stopping criteria is caused by the unsuitable BER threshold, which is obtained by estimating the expected BER performance at high SNRs, and this threshold does not indicate the correct termination according to convergence and non-convergence outputs (CNCO). Hence, in this paper, the threshold computation based on the BER of $\mathrm{CNCO}$ is proposed for an OBE stopping criterion (OBEsc). From the results, OBEsc is capable of terminating early in a varying SNR environment. The optimum number of iterations achieved by the OBEsc allows huge savings in decoding iteration number and decreasing the delay of turbo iterative decoding.
\end{abstract}

Keyword: Bit error rate; Estimation error; Stopping criterion; Turbo codes; Iterative decoding 